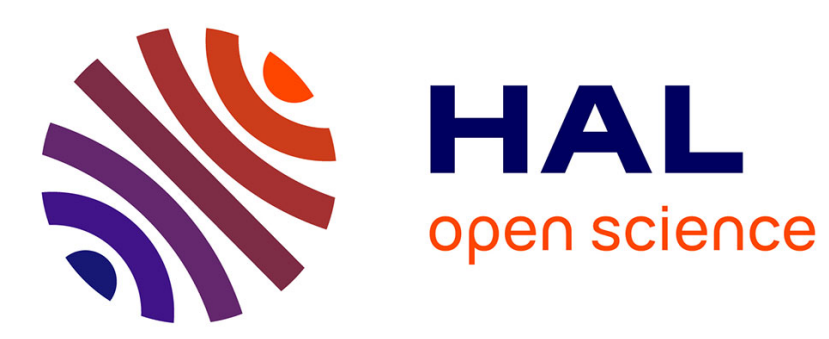

\title{
REACHABILITY OF FRACTIONAL DYNAMICAL SYSTEMS USING $\psi$-HILFER PSEUDO-FRACTIONAL DERIVATIVE
}

\author{
José Vanterler, M Vellappandi, V Govindaraj, Gastão Frederico
}

\section{- To cite this version:}

José Vanterler, M Vellappandi, V Govindaraj, Gastão Frederico. REACHABILITY OF FRACTIONAL DYNAMICAL SYSTEMS USING $\psi$-HILFER PSEUDO-FRACTIONAL DERIVATIVE. 2020. hal-02963296

\author{
HAL Id: hal-02963296 \\ https://hal.science/hal-02963296
}

Preprint submitted on 10 Oct 2020

HAL is a multi-disciplinary open access archive for the deposit and dissemination of scientific research documents, whether they are published or not. The documents may come from teaching and research institutions in France or abroad, or from public or private research centers.
L'archive ouverte pluridisciplinaire HAL, est destinée au dépôt et à la diffusion de documents scientifiques de niveau recherche, publiés ou non, émanant des établissements d'enseignement et de recherche français ou étrangers, des laboratoires publics ou privés. 


\title{
REACHABILITY OF FRACTIONAL DYNAMICAL SYSTEMS USING $\psi$-HILFER PSEUDO-FRACTIONAL DERIVATIVE
}

\author{
J. VAnterler dA C. Sousa* \\ Center for Mathematics, Computing and Cognition, Federal University of ABC \\ Avenida dos Estados, 5001, Bairro Bangu, 09.210-580, Santo André, SP - Brazil

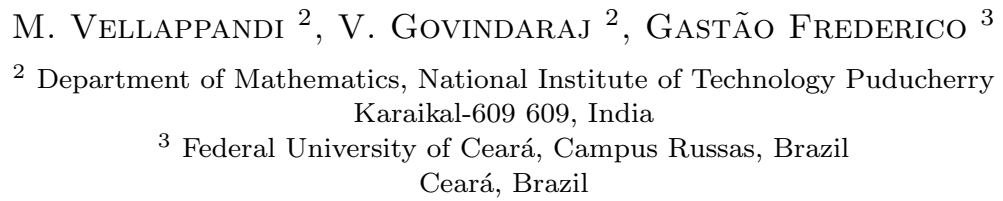 \\ (Communicated by the associate editor name)

\begin{abstract}
In this paper, we investigate the reachability of linear and nonlinear systems in the sense of the $\psi$-Hilfer pseudo-fractional derivative in $g$ calculus by means of the Mittag-Leffler functions (one and two parameters). In this sense, two numerical examples are discussed, in order to elucidate the investigated results.
\end{abstract}

1. Introduction. In this paper, we consider the linear fractional dynamical system governed by $\psi$-Hilfer fractional differential equation of the form

$$
\begin{aligned}
\mathbb{H}_{\oplus, \beta ;,+t_{0}+}^{\alpha, \beta} & =\mathbf{A} x(t) \oplus \mathbf{B} u(t), \quad t \in\left[t_{0}, t_{1}\right] \\
\mathbb{I}_{\oplus, \odot, t_{0}+}^{1-\gamma ; \psi} & x\left(t_{0}\right)=0,
\end{aligned}
$$

where $\mathbb{H}_{\oplus, \odot, t_{0}+}^{\alpha, \beta ;}(\cdot)$ is the $\psi$-Hilfer pseudo-fractional derivative of order $0<\alpha<1$ and type $0 \leq \beta \leq 1, \gamma=\alpha+\beta(1-\alpha), \mathbb{I}_{\oplus, \odot, t_{0}+}^{1-\gamma ;}(\cdot)$ is the Riemann-Liouville pseudofractional integral with respect to another function $1-\gamma$, the state vector $x \in \mathbb{R}^{n}$, the control vector $u \in \mathbb{R}^{m}$ and $\mathbf{A}$ and $\mathbf{B}$ are the constant matrices of dimension $n \times n$ and $n \times m$ respectively.

On the other hand, also in this paper, we consider the nonlinear fractional dynamical system governed by $\psi$-Hilfer fractional differential equation of the form

$$
\begin{aligned}
\mathbb{H}_{\oplus, \odot, t_{0}+}^{\alpha, \beta ; \psi} x(t) & =\mathbf{A} x(t) \oplus \mathbf{B} u(t) \oplus f(t, x(t), u(t)), \quad t \in\left[t_{0}, t_{1}\right] \\
\mathbb{I}_{\oplus, \odot ; \psi}^{1-\gamma, t_{0}+} x\left(t_{0}\right) & =0,
\end{aligned}
$$

where $\mathbb{H}_{\oplus, \odot, t_{0}+}^{\alpha, \beta ;}(\cdot)$ is the $\psi$-Hilfer pseudo-fractional derivative of order $0<\alpha<1$ and type $0 \leq \beta \leq 1, \gamma=\alpha+\beta(1-\alpha), \mathbb{I}_{\oplus, \odot, t_{0}+}^{1-\gamma ;}(\cdot)$ is the Riemann-Liouville pseudofractional integral with respect to another function $1-\gamma$, the state vector $x \in \mathbb{R}^{n}$,

2020 Mathematics Subject Classification. Primary: 26A33, 34A30, 34A34; Secondary: 47GXX, 93B03.

Key words and phrases. Fractional dynamical systems, reachability Grammian, $\psi$-Hilfer pseudo-fractional derivative.

* Corresponding author: J. Vanterler da C. Sousa. 
the control vector $u \in \mathbb{R}^{m}$ and $\mathbf{A}$ and $\mathbf{B}$ are the constant matrices of dimension $n \times n$ and $n \times m$ respectively and the nonlinear function $f:\left[t_{0}, t_{1}\right] \times \mathbb{R}^{n} \times \mathbb{R}^{m} \rightarrow \mathbb{R}^{n}$ is continuous.

One of the central questions of these last years, has been the study and advancement of the theory of fractional differential equations and applications [25, 29, 3, 42, $15,7,40,38]$. On the one hand, the growing number of researchers in the field and the importance and relevance of the different types of fractional differential equations, has gained prominence and attention in the scientific community. One of the main causes of discussing properties of solutions of fractional differential equations is due to the fact that the fractional case allows us to perform certain analyzes that the whole case does not, because the order of the differentiation operator varies $n-1<\alpha<n$, and in particular, holds the entire case when $\alpha=1$. A priory this is one of the first and main purpose. After that, each problem deserves its attention to the determined objective that is investigated.

In mid 2018, Sousa and Oliveira [36, 37], introduced the so-called $\psi$-Hilfer fractional derivative, motivated by the simple problem of knowing which fractional derivative to use to discuss differential equation problems, because until then a wide class of fractional derivatives exists. In this sense, since the $\psi$-Hilfer fractional derivative is a global and general operator, which contains a wide class of particular cases, numerous researchers began to discuss problems of differential equations via this derivative, from existence and uniqueness properties, until the attractiveness of solutions $[4,2,13,11,27,8,39,10]$. In this sense, motivated by these questions and problems still open in $g$-calculus, in 2020 Sousa et al. [41], extended the $\psi$-Hilfer fractional derivative to the context of $g$-calculus. In this area involving $g$-calculus and fractional calculus, there are few jobs and open questions need to be answered.

Reachability is the most important fundamental concepts in mathematical control theory and it is one of the structural property of dynamical systems. Roughly speaking, reachability generally means, that it is possible to steer the dynamical system from the zero initial state to an arbitrary final state by using the set of admissible input functions. In 2008 Trzasko [35], considered a fractional positive linear problem in discrete time systems with delay described by the state equations. In this sense, they discussed necessary and sufficient conditions are established for the positivity, reachability and controllability to zero for fractional systems with one delay in state. Recently, Kaczorek introduced a new class of fractional positive continuous-time linear system and investigate the reachability of positive fractional system in [18] and addressed the relationship between the reachability of positive continuous-time linear system and positive standard system in [25]. Kociszewski [28] proposed the reachability of fractional discrete-time linear systems with delays in state and control. Similarly, reachability for the fractional discrete-time linear systems with multiple delays in state was investigated by Buslowicz in [9].

In 2013, Zhang et al. [44], discussed the reachability and controllability of the following fractional singular dynamical systems with control delay

$$
\begin{aligned}
E^{c} D^{\alpha} x(t) & =A x(t)+B u(t)+C u(t-\tau), \quad t \geq 0 \\
u(t) & =\psi(t), \quad-\tau \leq t \leq 0 \\
u(0) & =x_{0},
\end{aligned}
$$

where $D^{\alpha} x(t)$ denotes an $\alpha$ order Caputo fractional derivative of $x(t)$, and $0<$ $\alpha \leq 1 ; E, A, B$ and $C$ are the known constant matrices, $E, A \in \mathbb{R}^{n \times n}, B \in \mathbb{R}^{n \times m}$, 
$C \in \mathbb{R}^{n \times m}$, and rank $(E<n) ; x \in \mathbb{R}^{n}$ is the state variable; $u \in \mathbb{R}^{m}$ is the control imput; $\tau>0$ is the time control delay; and $\psi(t)$ is the initial control function.

A few years later, Sajewski [33], discussed reachability, observability and minimum energy control problems for the fractional positive continuous-time linear systems with two different fractional orders are formulated. Necessary and sufficient conditions for the reachability and observability are established. Solution to the minimum energy control problem is derived and demonstrated on example of electrical circuit. For further research, the interested reader can refer to $[23,19,20,26,24,21]$ and references there in.

On the other hand, problems involving controllability and observability of solutions to fractional differential equations have also been the target of research in the scientific community. Other qualitative properties of solutions of fractional differential equations can be obtained in means of scientific dissemination [30, 43, 29, 22, $6,5,14]$.

There are few studies in the literature involving reachability of fractional differential equation problems. This becomes more restricted when it comes to fractional differential equations in the sense of the $\psi$-Hilfer pseudo-fractional derivative, for several reasons. The first is due to the fact that the operator was recently introduced by Sousa et al. [41]. Second, there are still no works to be done involving the existence and uniqueness of solutions of fractional differential equations. In this sense, we are providing one of the first work on the reachability of linear and nonlinear fractional dynamical systems.

So motivated by the works above, and by innumerable open questions in this area, in particular, because it is a starting point of this issue involving the $\psi$-Hilfer pseudofractional operator, we present clearly and lucidly what were the main objectives discussed and achieved in this article. In this sense, we have:

- We present a new class of mild solutions for a fractional differential equation problem in the sense of the $\psi$-Hilfer pseudo-fractional derivative in terms of the one and two-parameter Mittag-Leffler function.

- We discuss the reachability of the linear fractional dynamical system given by $\psi$-Hilfer fractional differential equation (see Eq.(1)).

- We discuss the reachability of the nonlinear fractional dynamical system given by $\psi$-Hilfer fractional differential equation (see Eq.(2)).

- We present two numerical examples and plot their respective graphs, in order to elucidate the results discussed in this paper.

In the rest, the article is divided as follows. In section 2 , we present some definitions and essential results for the development of the article, in particular, we discuss the mild solution for a differential equation in the sense of the $\psi$-Hilfer pseudo-fractional derivative in terms of the Mittag-Leffler function. In section 3, we discussed the reachability of the linear fractional dynamical system given by $\psi$ Hilfer fractional differential equation. In section 4, we investigated reachability of the nonlinear fractional dynamical system given by $\psi$-Hilfer fractional differential equation. Finally, we conclude the article with two examples and their respective graphs in order to elucidate the results obtained in the article.

\section{Preliminaries.}

Definition 2.1. [1, 17, 31, 32] A binary operator $\oplus$ on $[a, b]$ is pseudo-addition if it is commutative, non-decreasing, with respect to $\preceq$, continuous; associative, and with a zero (neutral) element denoted by 0 . Let $[a, b]_{+}=\{x \mid, x \in[a, b], 0 \leq x\}$. 
4. VANTERLER DA C. SOUSA, M. VELlAPPANDI, V. GOVINDARAJ AND GASTÃO S. F. FREDERICO.

Definition 2.2. $[1,17,31,32]$ A binary operation $\odot$ on $[a, b]$ is pseudo-multiplication if it is commutative, positively non-decreasing, i.e., $x \leq y$ implies $x \odot z \leq y \odot z$ for all $z \in[a, b]_{+}$, associative and with a unit element $1 \in[a, b]$, i.e., for each $x \in[a, b], 1 \odot x=x$. Also, $0 \odot x=0$ and that $\odot$ is distributive over $\oplus$, i.e., $x \odot(y \oplus z)=(x \odot y) \oplus(x \odot) z$.

The structure $([a, b], \oplus, \odot)$ is a semiring $[1,17,31,32]$.

Definition 2.3. [1, 17, 31, 32] An important class of pseudo-operations $\oplus$ and $\odot$ is when these are defined by a monotone and continuous function $g:[a, b] \rightarrow[0, \infty]$, i.e., pseudo-operations $\oplus$ and $\odot$ are given by

$$
x \oplus y=g^{-1}(g(x)+g(y)) \text { and } x \odot y=g^{-1}(g(x) g(y)) .
$$

Definition 2.4. $[1,17,31,32]$ Let $X$ be a non-empty set and $\mathcal{A}$ be a $\sigma$-algebra of subsets of a set $X$. A set function $\mu: \mathcal{A} \rightarrow[a, b]$ is called a $\sigma$ - $\oplus$-measure if the following conditions are satisfy:

1. $\mu(\emptyset)=0$;

2. $\mu\left(\bigcup_{i=1}^{\infty} \mathcal{A}_{i}\right)=\bigoplus_{i=1}^{\infty} \mu\left(\mathcal{A}_{i}\right)$

holds for any sequence $\{\mathcal{A}\}_{i \in \mathbb{N}}$ of pairwise disjoint sets from $\mathcal{A}$.

Definition 2.5. $[1,17,31,32]$ Let pseudo-operations $\oplus$ and $\odot$ be defined a monotone and continuous function $g:[a, b] \rightarrow[0, \infty]$.

1. The $g$-integral for a measurable function $f:[c, d] \rightarrow[a, b]$ is given by

$$
\int_{[c, d]}^{\oplus} f \odot \mathrm{d} x=g^{-1}\left(\int_{c}^{d} g(f(x)) \mathrm{d} x\right) .
$$

2. The $g$-Laplace of a function $f$ is defined by

$$
\mathscr{L}^{\oplus}[f(x)]=g^{-1}(\mathscr{L}[g(f(x))]) .
$$

Definition 2.6. $[1,17,31,32]$ Let $g$ be the additive generator of the strict-pseudoaddition $\oplus$ on $[a, b]$ such that $g$ is continuous differentiable on $(a, b)$. The corresponding pseudo-multiplication $\odot$ will always be defined as $u \odot v=g^{-1}(g(u) \cdot g(v))$. If the function $f$ is differentiable on $(c, d)$ and has the same monotonicity as the function $g$, then the $g$-derivative of $f$ at the point $x \in(c, d)$ is defined by

$$
\frac{\mathrm{d}^{\oplus}}{\mathrm{d} x} f(x)=g^{-1}\left(\frac{\mathrm{d}}{\mathrm{d} x} g(f(x))\right) .
$$

Also, if there exists the $n$ - $g$-derivative of $f$, then

$$
\frac{\mathrm{d}^{(n) \oplus}}{\mathrm{d} x} f(x)=g^{-1}\left(\frac{\mathrm{d}^{n}}{\mathrm{~d} x^{n}} g(f(x))\right) .
$$

Definition 2.7. $[1,17,31,32]$ Let $g$ be a generator of a pseudo-addition $\oplus$ on interval $[-\infty,+\infty]$. Binary operation $\ominus$ and $\oslash$ on $[-\infty,+\infty]$ are defined by the expressions

$$
x \ominus y=g^{-1}(g(x)-g(y)) \quad \text { and } \quad x \oslash y=g^{-1}\left(\frac{g(x)}{g(y)}\right) .
$$

If the expressions $g(x)-g(y)$ and $\frac{g(x)}{g(y)}$ have sense are said to be the pseudosubtraction and pseudo-division consistent with the pseudo-addition $\oplus$. 
Definition 2.8. $[1,17,31,32]$ Let $g:[-\infty,+\infty] \rightarrow[-\infty,+\infty]$ be a continuous, strictly increasing and odd function such that $g(0)=0, g(1)=1$ and $g(+\infty)=$ $+\infty$. The system of pseudo-arithmetical operations $\{\oplus, \oslash, \odot, \ominus\}$ generated by these functions is said to be the consistent system.

Definition 2.9. [36, 37] $[\psi$-Riemann-Liouville fractional integral] Let $I=(a, b)$ $(-\infty \leq a<b \leq \infty)$ be a finite or infinite interval of the real line $\mathbb{R}$ and $\alpha>0$. Also let $\psi(x)$ be an increasing and positive monotone function on $(a, b]$, having a continuous derivative $\psi^{\prime}(x)$ on $I$. The left-sided and right-sided fractional integrals of a function $f$ with respect to another function $\psi$ on $J=[a, b]$ are defined by

$$
\mathcal{I}_{a+}^{\alpha ; \psi} f(x)=\frac{1}{\Gamma(\alpha)} \int_{a}^{x} \psi^{\prime}(t)(\psi(x)-\psi(t))^{\alpha-1} f(t) d t
$$

and

$$
\mathcal{I}_{b-}^{\alpha ; \psi} f(x)=\frac{1}{\Gamma(\alpha)} \int_{x}^{b} \psi^{\prime}(t)(\psi(t)-\psi(x))^{\alpha-1} f(t) d t .
$$

Definition 2.10. [36, 37][ $\psi$-Hilfer fractional derivative] Let $n-1<\alpha<n$, with $n \in \mathbb{N}, J$ is an interval such that $-\infty \leq a<b \leq+\infty$ and $f, \psi \in \mathcal{C}^{n}(J, \mathbb{R})$ are two functions such that $\psi$ is increasing and $\psi(t) \neq 0$, for all $t \in J$. The $\psi$ Hilfer fractional derivative left-sided and right-sided, denoted by ${ }^{H} \mathbb{D}_{a^{+}}^{\alpha, \beta ; \psi}(\cdot)$ and ${ }^{H} \mathbb{D}_{b^{-}}^{\alpha, \beta ; \psi}(\cdot)$ of a function $f$ of order $\alpha$ and type $0 \leq \beta \leq 1$, is defined by

$$
{ }^{H} \mathbb{D}_{a^{+}}^{\alpha, \beta ; \psi} f(x)=\mathcal{I}_{a^{+}}^{\beta(n-\alpha) ; \psi}\left(\frac{1}{\psi^{\prime}(x)} \frac{d}{d x}\right)^{n} \mathcal{I}_{a^{+}}^{(1-\beta)(n-\alpha) ; \psi} f(x)
$$

and

$$
{ }^{H} \mathbb{D}_{b^{-}}^{\alpha, \beta ; \psi} f(x)=\mathcal{I}_{b^{-}}^{\beta(n-\alpha) ; \psi}\left(-\frac{1}{\psi^{\prime}(x)} \frac{d}{d x}\right)^{n} \mathcal{I}_{b^{-}}^{(1-\beta)(n-\alpha) ; \psi} f(x)
$$

where $\mathcal{I}_{a^{+}}^{\alpha ; \psi}(\cdot)$ and $\mathcal{I}_{b^{-}}^{\alpha ; \psi}(\cdot)$ by defined in (27) and (28) respectively.

Definition 2.11. [41, 1] ( $\psi$-Riemann-Liouville pseudo-fractional integral) Let a generator $g: J \rightarrow[0, \infty]$ of the pseudo-addition $\oplus$ and the pseudo-multiplication $\odot$ be an increasing function. Also let $\psi$ be an increasing and positive function on $(a, b]$, having a continuous derivative $\psi^{\prime}(x)$ on $I$. The left-sided and the right-sided $\psi$-Riemann-Liouville pseudo-fractional integrals of order $\alpha>0$ of a measurable function $f: J \rightarrow J$ with respect to function $\psi$ on $J$ are defined by:

$$
\begin{aligned}
\mathbb{I}_{\oplus, \odot, a+}^{\alpha ; \psi} f(x) & =g^{-1}\left(\mathcal{I}_{a+}^{\alpha ; \psi} g(f(x))\right) \\
& =\int_{[a, x]}^{\oplus}\left[g^{-1}\left(\frac{\psi^{\prime}(t)(\psi(x)-\psi(t))^{\alpha-1}}{\Gamma(\alpha)}\right) \odot f(t)\right] \odot d t
\end{aligned}
$$

and

$$
\begin{aligned}
\mathbb{I}_{\oplus, \odot, b-}^{\alpha ; \psi} f(x) & =g^{-1}\left(\mathcal{I}_{b-}^{\alpha ; \psi} g(f(x))\right) \\
& =\int_{[x, b]}^{\oplus}\left[g^{-1}\left(\frac{\psi^{\prime}(t)(\psi(t)-\psi(x))^{\alpha-1}}{\Gamma(\alpha)}\right) \odot f(t)\right] \odot d t,
\end{aligned}
$$

where $\mathcal{I}_{a+}^{\alpha ; \psi}(\cdot)$ and $\mathcal{I}_{b-}^{\alpha ; \psi}(\cdot)$ are given by Eq.(27) and Eq.(28), respectively. 
6. VANTERlER DA C. SOUSA, M. VELlAPPANDI, V. GOVINDARAJ AND GASTÃO S. F. FREDERICO.

Definition 2.12. [41] ( $\psi$-Hilfer pseudo-fractional derivative) Let a generator $g$ : $[a, b] \rightarrow[0, \infty]$ of the pseudo-addition $\oplus$ and the pseudo-multiplication $\odot$ be an increasing function. Also let $\psi \in C^{n}([a, b], \mathbb{R})$, a function such that $\psi$ be an increasing and positive function on $(a, b]$ having a continuous derivative $\psi^{\prime}$ on $(a, b)$ and $\psi^{\prime} \neq 0$ for all $x \in[a, b]$. The left-sided and right-sided $\psi$-Hilfer pseudo-fractional derivative of order $n-1<\alpha<n$ and type $0 \leq \beta \leq 1$, of a measurable function $f:[a, b] \rightarrow[a, b]$ is defined by

$$
\begin{aligned}
\mathbb{H}_{\oplus, \odot, a+}^{\alpha, \beta ; \psi} f(x) & =g^{-1}\left({ }^{H} \mathbb{D}_{a+}^{\alpha, \beta ; \psi} g(f(x))\right) \\
& =\mathbb{I}_{\oplus, \odot, a+}^{\beta(n-\alpha) ; \psi} g^{-1}\left(\left(\frac{D}{\psi^{\prime}(x)}\right)^{n}\right) \odot \mathbb{I}_{\oplus, \odot, a+}^{1-\gamma ; \psi} f(x)
\end{aligned}
$$

and

$$
\begin{aligned}
\mathbb{H}_{\oplus, \odot, b-}^{\alpha, \beta ; \psi} f(x) & =g^{-1}\left({ }^{H} \mathbb{D}_{b-}^{\alpha, \beta ; \psi} g(f(x))\right) \\
& =\mathbb{I}_{\oplus, \odot, b-}^{\beta(n-\alpha) ; \psi} g^{-1}\left(\left(-\frac{D}{\psi^{\prime}(x)}\right)^{n}\right) \odot \mathbb{I}_{\oplus, \odot, b-}^{1-\gamma ; \psi} f(x),
\end{aligned}
$$

where ${ }^{H} \mathbb{D}_{a+}^{\alpha, \beta ; \psi}(\cdot)$ and ${ }^{H} \mathbb{D}_{b-}^{\alpha, \beta ; \psi}(\cdot)$ are $\psi$-Hilfer fractional derivative are given by Eq.(29) and Eq.(30).

Note that

$$
\begin{aligned}
& \mathbb{H}_{\oplus, \odot, a+}^{\alpha, \beta ; \psi} f(x)=g^{-1}\left(\mathcal{I}_{a+}^{\gamma-\alpha ; \psi} R L_{\mathbb{D}_{a+}^{\gamma ; \psi}}^{\gamma(f}(f(x))\right) \\
& =\mathbb{I}_{\oplus, \odot, a+}^{\gamma-\alpha ; \psi} R L \mathbb{D}_{\oplus, \odot, a+}^{\gamma ; \psi} f(x)
\end{aligned}
$$

and

$$
\begin{aligned}
\mathbb{H}_{\oplus, \odot, b-}^{\alpha, \beta ; \psi} f(x) & =g^{-1}\left(\mathcal{I}_{b-}^{\gamma-\alpha ; \psi} R L_{\mathbb{D}_{b-}^{\gamma ; \psi}}^{\gamma} g(f(x))\right) \\
& =\mathbb{I}_{\oplus, \odot, b-}^{\gamma-\alpha ; \psi} R \mathbb{D}_{\oplus, \odot, b-}^{\gamma ; \psi} f(x),
\end{aligned}
$$

where $\gamma=\alpha+\beta(n-\alpha)$.

Theorem 2.13. [41] Let $f:[a, b] \rightarrow[a, b]$ be a measurable functions. If $n \in \mathbb{N}$, then we have

1. $\mathbb{H}_{\oplus, \odot, a+}^{0, \beta ; \psi} f(x)=f(x)$.

2. $\mathbb{H}_{\oplus, \odot, a+}^{1, \beta ; \psi} f(x)=g^{-1}\left(\left(\frac{D}{\psi^{\prime}(x)}\right) g(f(x))\right)$.

3. $\mathbb{H}_{\oplus, \odot, a+}^{n, \beta ; \psi} f(x)=\left(\frac{D}{\psi^{\prime}(x)}\right)^{(n) \oplus} f(x)$.

4. $\mathbb{H}_{\oplus, \odot, a+}^{\alpha, \beta ; \psi}\left(f_{1}(x) \oplus f_{2}(x)\right)=\mathbb{H}_{\oplus, \odot, a+}^{\alpha, \beta ; \psi} f_{1}(x) \oplus \mathbb{H}_{\oplus, \odot, a+}^{\alpha, \beta ; \psi} f_{2}(x)$.

5. $\mathbb{H}_{\oplus, \odot, a+}^{\alpha, \beta ; \psi}(\lambda \odot f(x))=\lambda \odot \mathbb{H}_{\oplus, \odot, a+}^{\alpha, \beta ; \psi} f(x)$.

6. $\mathbb{H}_{\oplus, \odot, a+}^{\alpha, \beta ; \psi} \mathbb{I}_{\oplus, \odot, a+}^{\alpha ; \psi} f(x)=f(x)$.

7. $\mathbb{I}_{\oplus, \odot, a+}^{\alpha ; \psi} \mathbb{H}_{\oplus, \odot, a+}^{\alpha, \beta ; \psi} f(x)=f(x) \ominus\left[\bigoplus_{k=1}^{n} g^{-1}\left(C_{k}\right) \odot g^{-1}(\psi(x)-\psi(a))^{\gamma-k}\right]$ with

$$
\gamma=\alpha+\beta(n-\alpha) \text { and } C_{k}=\frac{(g \circ f)^{[n-k]} \mathbb{I}_{\oplus, \odot, \gamma ; a+}^{n-\gamma ;} g(f(a))}{\Gamma(\gamma-k+1)} .
$$


Definition 2.14. Let $n \in \mathbb{N}$. Then we can consider the following sequential $\psi$ fractional derivative for suitable functions $y(x)$

$$
\begin{aligned}
y_{\oplus}^{(k \alpha), \beta ; \psi}(x) & =g^{-1}\left(D^{k \alpha, \beta ; \psi} g(y(x))\right) \\
& =g^{-1}\left(D^{\alpha, \beta ; \psi} D^{(k-1) \alpha, \beta ; \psi} g(y(x))\right),
\end{aligned}
$$

where $k=1, \ldots, n, D^{0} y(x)=y(x)$, and $D^{\alpha, \beta ; \psi}$ is any fractional differential operator, for example, it could be ${ }^{H} \mathbb{D}_{a+}^{\alpha, \beta ; \psi}$.

Now, we have

$$
\mathbb{I}_{\oplus, \odot, a+}^{\alpha ; \psi} f(0)=g^{-1}\left(\lim _{x \rightarrow a} \psi(x)^{1-\alpha} g(f(x))\right) .
$$

Definition 2.15. [36] The Mittag-Leffler function $\mathbb{E}_{\alpha, \beta}(z)$ is a complex function which depends on two complex parameter, and it is defined by

$$
\mathbb{E}_{\alpha, \beta}(z)=\sum_{k=0}^{\infty} \frac{z^{k}}{\Gamma(\alpha k+\beta)}, \alpha, \beta>0 .
$$

The function $\mathbb{E}_{\alpha, \beta}$ converges for all values of the argument $z$. For a $n \times n$ matrix $\mathbf{A}$, the matrix extension of the above Mittag-Leffler function is

$$
\mathbb{E}_{\alpha, \beta}(\mathbf{A})=\sum_{k=0}^{\infty} \frac{\mathbf{A}^{k}}{\Gamma(\alpha k+\beta)} .
$$

In general $\mathbb{E}_{\alpha, 1}(\mathbf{A})=\mathbb{E}_{\alpha, \beta}(A)$. If $\alpha>0$ and $\mathbf{A}$ is $n \times n$ matrix, then

$$
\mathbb{H}_{\oplus, \odot, a+}^{\alpha, \beta ; \psi} \mathbb{E}_{\alpha, \beta}\left(\mathbf{A}(\psi(x)-\psi(a))^{\alpha}\right)=\mathbf{A} \mathbb{E}_{\alpha, \beta}\left(A(\psi(x)-\psi(a))^{\alpha}\right) .
$$

Consider the following $\psi$-Hilfer fractional differential equation

$$
\left\{\begin{array}{l}
\mathbb{H}_{\oplus, \beta ; \psi, t_{0}+}^{\alpha, \beta(t)}=\mathbf{A} x(t) \oplus f(t), t \in\left[t_{0}, t_{1}\right] \\
\mathbb{I}_{\oplus, \odot ;, t_{0}+}^{-\gamma\left(t_{0}\right)}=x_{0}
\end{array}\right.
$$

Applying the operator $\mathbb{I}_{\oplus, \odot ; t_{0}+}^{1-\gamma ; \psi}(\cdot)$ on both sides of the Eq.(19) and using the Theorem 2.13, one has $x(t) \ominus\left[g^{-1}\left(C_{1}\right) \odot g^{-1}(\psi(x)-\psi(a))^{\gamma-k}\right]=\mathbb{I}_{\oplus, \odot, t_{0}+}^{1-\gamma ; \psi}(\mathbf{A} x(t) \oplus f(t))$. This implies

$x(t)=g^{-1}\left(C_{1}\right) \odot g^{-1}\left(\psi(t)-\psi\left(t_{0}\right)\right)^{\gamma-k} \oplus \int_{\left[t_{0}, t\right]}^{\oplus} g^{-1}\left(\frac{\psi^{\prime}(s)(\psi(t)-\psi(s))^{\alpha-1}}{\Gamma(\alpha)}\right) \odot(\mathbf{A} x(s) \oplus f(s)) \odot d s$,

where $C_{1}=\frac{I_{t_{0}+}^{1-\gamma ; \psi} g(x(t))}{\Gamma(\gamma)}$.

Note that, the Eq.(20) can be rewritten as of the form

$$
x(t)=\frac{\mathbb{I}_{\oplus, \odot, t_{0}+}^{1-\gamma ; \psi}\left(t_{0}\right)}{\Gamma(\gamma)} \odot g^{-1}\left(\psi(t)-\psi\left(t_{0}\right)\right)^{\gamma-k} \oplus \int_{\left[t_{0}, t\right]}^{\oplus} g^{-1}\left(\frac{\psi^{\prime}(s)(\psi(t)-\psi(s))^{\alpha-1}}{\Gamma(\alpha)}\right) \odot(\mathbf{A} x(s) \oplus f(s)) \odot d s .
$$

Now, through successive approximations, let's get an expression for Eq.(21).

Let for this set

$$
x_{0}(t)=\frac{x_{0}}{\Gamma(\gamma)} \odot g^{-1}\left(\psi(t)-\psi\left(t_{0}\right)\right)^{\gamma-1}
$$


8. VANTERlER DA C. SOUSA, M. VELlappandi, V. GOVINDARAJ AND GASTÃo S. F. FREDERICO. and

$$
\begin{aligned}
x_{m}(t)= & \frac{x_{0}}{\Gamma(\gamma)} \odot g^{-1}\left(\psi(t)-\psi\left(t_{0}\right)\right)^{\gamma-1} \\
& \oplus \int_{\left[t_{0}, t\right]}^{\oplus} g^{-1}\left(\frac{\psi^{\prime}(s)(\psi(t)-\psi(s))^{\alpha-1}}{\Gamma(\alpha)}\right) \odot\left(\mathbf{A} x_{m-1}(s) \oplus f(s)\right) \odot d s .
\end{aligned}
$$

Using the Eq.(22) and Eq.(23), we find

$$
\begin{aligned}
& x_{1}(t)=x_{0}(t) \oplus \int_{\left[t_{0}, t\right]}^{\oplus} g^{-1}\left(\frac{\psi^{\prime}(s)(\psi(t)-\psi(s))^{\alpha-1}}{\Gamma(\alpha)}\right) \odot\left(\mathbf{A} x_{0}(s) \oplus f(s)\right) \odot d s \\
& =\frac{x_{0}}{\Gamma(\gamma)} \odot g^{-1}\left(\psi(t)-\psi\left(t_{0}\right)\right)^{\gamma-1} \oplus \int_{\left[t_{0}, t\right]}^{\oplus} g^{-1}\left(\frac{\psi^{\prime}(s)(\psi(t)-\psi(s))^{\alpha-1}}{\Gamma(\alpha)}\right) \\
& \odot\left(\mathbf{A} \frac{x_{0}}{\Gamma(\gamma)} \odot g^{-1}\left(\psi(s)-\psi\left(t_{0}\right)\right)^{\gamma-1} \oplus f(s)\right) \odot d s \\
& =\frac{x_{0}}{\Gamma(\gamma)} \odot g^{-1}\left(\psi(t)-\psi\left(t_{0}\right)\right)^{\gamma-1} \oplus \\
& \mathbf{A} \frac{x_{0}}{\Gamma(\gamma)} \odot \int_{\left[t_{0}, t\right]}^{\oplus} g^{-1}\left(\frac{\psi^{\prime}(s)(\psi(t)-\psi(s))^{\alpha-1}}{\Gamma(\alpha)}\right) \odot g^{-1}\left(\psi(s)-\psi\left(t_{0}\right)\right)^{\gamma-1} \odot d s \\
& \oplus \int_{\left[t_{0}, t\right]}^{\oplus} g^{-1}\left(\frac{\psi^{\prime}(s)(\psi(t)-\psi(s))^{\alpha-1}}{\Gamma(\alpha)}\right) \odot f(s) \odot d s \\
& =\frac{x_{0}}{\Gamma(\gamma)} \odot g^{-1}\left(\psi(t)-\psi\left(t_{0}\right)\right)^{\gamma-1} \oplus \mathbf{A} \frac{x_{0}}{\Gamma(\gamma)} \odot \mathbb{I}_{\oplus, \odot, t_{0}+}^{\alpha ; \psi}\left(g^{-1}\left(\psi(t)-\psi\left(t_{0}\right)\right)^{\gamma-1}\right) \\
& \oplus \mathbb{I}_{\oplus, \odot, t_{0}+}^{\alpha ; \psi} f(t) \text {. }
\end{aligned}
$$


On the other hand, we have

$$
\begin{aligned}
& x_{2}(t)=\frac{x_{0}}{\Gamma(\gamma)} \odot g^{-1}\left(\psi(t)-\psi\left(t_{0}\right)\right)^{\gamma-1} \oplus \int_{\left[t_{0}, t\right]}^{\oplus} g^{-1}\left(\frac{\psi^{\prime}(s)(\psi(t)-\psi(s))^{\alpha-1}}{\Gamma(\alpha)}\right) \\
& \odot\left(\mathbf{A} x_{1}(s) \oplus f(s)\right) \odot d s \\
& =\frac{x_{0}}{\Gamma(\gamma)} \odot g^{-1}\left(\psi(t)-\psi\left(t_{0}\right)\right)^{\gamma-1} \oplus \int_{\left[t_{0}, t\right]}^{\oplus} g^{-1}\left(\frac{\psi^{\prime}(s)(\psi(t)-\psi(s))^{\alpha-1}}{\Gamma(\alpha)}\right) \\
& \odot \mathbf{A} \frac{x_{0}}{\Gamma(\gamma)} \odot g^{-1}\left(\psi(s)-\psi\left(t_{0}\right)\right)^{\gamma-1} \odot d s \\
& \oplus \int_{\left[t_{0}, t\right]}^{\oplus} g^{-1}\left(\frac{\psi^{\prime}(s)(\psi(t)-\psi(s))^{\alpha-1}}{\Gamma(\alpha)}\right) \odot \mathbf{A}^{2} \frac{x_{0}}{\Gamma(\gamma)} \odot \mathbb{I}_{\oplus, \odot, t_{0+}}^{\alpha ; \psi} g^{-1}\left((\psi(t)-\psi(s))^{\gamma-1}\right) \odot d s \\
& \oplus \int_{\left[t_{0}, t\right]}^{\oplus} g^{-1}\left(\frac{\psi^{\prime}(s)(\psi(t)-\psi(s))^{\alpha-1}}{\Gamma(\alpha)}\right) \odot \mathbf{A} \odot \mathbb{I}_{\oplus, \odot, t_{0+}}^{\alpha ; \psi} f(s) \odot d s \\
& \oplus \int_{\left[t_{0}, t\right]}^{\oplus} g^{-1}\left(\frac{\psi^{\prime}(s)(\psi(t)-\psi(s))^{\alpha-1}}{\Gamma(\alpha)}\right) \odot f(s) \odot d s \\
& =x_{0} \odot \int_{\left[t_{0}, t\right]}^{\oplus} \sum_{k=0}^{2} \mathbf{A}^{k} \odot g^{-1}\left(\frac{\psi^{\prime}(s)(\psi(t)-\psi(s))^{\alpha k-1}}{\Gamma(\alpha k)}\right) \frac{g^{-1}\left(\left(\psi(s)-\psi\left(t_{0}\right)\right)^{\gamma-1}\right)}{\Gamma(\gamma)} \odot d s \\
& \oplus \int_{\left[t_{0}, t\right]}^{\oplus} \sum_{k=1}^{2} \mathbf{A}^{k} \odot g^{-1}\left(\frac{\psi^{\prime}(s)(\psi(t)-\psi(s))^{\alpha k-1}}{\Gamma(\alpha k)}\right) \odot f(s) \odot d s .
\end{aligned}
$$

Continuing this process, we derive the following relation

$$
\begin{aligned}
x_{m}(t)= & x_{0} \odot \int_{\left[t_{0}, t\right]}^{\oplus} \sum_{k=0}^{m} \mathbf{A}^{k} \odot g^{-1}\left(\frac{\psi^{\prime}(s)(\psi(t)-\psi(s))^{\alpha k-1}}{\Gamma(\alpha k)}\right) \frac{g^{-1}\left(\left(\psi(s)-\psi\left(t_{0}\right)\right)^{\gamma-1}\right)}{\Gamma(\gamma)} \odot d s \\
& \oplus \int_{\left[t_{0}, t\right]}^{\oplus} \sum_{k=0}^{m} \mathbf{A}^{k} \odot g^{-1}\left(\frac{\psi^{\prime}(s)(\psi(t)-\psi(s))^{\alpha k+\alpha-1}}{\Gamma(\alpha k+\alpha)}\right) \odot f(s) \odot d s
\end{aligned}
$$

Taking limit as $m \rightarrow \infty$ on both sides of the Eq.(25), we have

$$
\begin{aligned}
\lim _{m \rightarrow \infty} x_{m}(t)= & x_{0} \odot \int_{\left[t_{0}, t\right]}^{\oplus} \sum_{k=0}^{\infty} \mathbf{A}^{k} \odot g^{-1}\left(\frac{\psi^{\prime}(s)(\psi(t)-\psi(s))^{\alpha k-1}}{\Gamma(\alpha k+\gamma)}\right) g^{-1}\left(\left(\psi(s)-\psi\left(t_{0}\right)\right)^{\gamma-1}\right) \odot d s \\
& \oplus \int_{\left[t_{0}, t\right]}^{\oplus} \sum_{k=0}^{\infty} \mathbf{A}^{k} \odot g^{-1}\left(\frac{\psi^{\prime}(s)(\psi(t)-\psi(s))^{\alpha k+\alpha-1}}{\Gamma(\alpha k+\alpha)}\right) \odot f(s) \odot d s \\
= & x_{0} \odot g^{-1}\left(\left(\psi(t)-\psi\left(t_{0}\right)\right)^{\gamma-1}\right) \odot \int_{\left[t_{0}, t\right]}^{\oplus} g^{-1}\left(\psi^{\prime}(s)\right) \odot \mathbb{E}_{\alpha, \gamma}\left(g(\mathbf{A})(\psi(t)-\psi(s))^{\alpha}\right) \odot d s \\
& \oplus \int_{\left[t_{0}, t\right]}^{\oplus} g^{-1}\left(\psi^{\prime}(s)(\psi(t)-\psi(s))^{\alpha-1}\right) \odot \mathbb{E}_{\alpha, \alpha}\left(g(\mathbf{A})(\psi(t)-\psi(s))^{\alpha}\right) \odot f(s) \odot d s .
\end{aligned}
$$


IO VANTERLER DA C. SOUSA, M. VELLAPPANDI, V. GOVINDARAJ AND GASTÃO S. F. FREDERICO.

Therefore, we conclude that

$$
\begin{aligned}
x(t)= & x_{0} \odot g^{-1}\left(\left(\psi(t)-\psi\left(t_{0}\right)\right)^{\gamma-1}\right) \odot \int_{\left[t_{0}, t\right]}^{\oplus} g^{-1}\left(\psi^{\prime}(s)\right) \odot \mathbb{E}_{\alpha, \gamma}\left(g(\mathbf{A})(\psi(t)-\psi(s))^{\alpha}\right) \odot d s \\
& \oplus \int_{\left[t_{0}, t\right]}^{\oplus} g^{-1}\left(\psi^{\prime}(s)(\psi(t)-\psi(s))^{\alpha-1}\right) \odot \mathbb{E}_{\alpha, \alpha}\left(g(\mathbf{A})(\psi(t)-\psi(s))^{\alpha}\right) \odot f(s) \odot d s .
\end{aligned}
$$

The particular important case of the Eq.(26) is given when taking $g(x)=x$,

$$
\begin{aligned}
x(t)= & x_{0}\left(\psi(t)-\psi\left(t_{0}\right)\right)^{\gamma-1} \mathbb{E}_{\alpha, \gamma}\left(\mathbf{A}(\psi(t)-\psi(s))^{\alpha}\right) \\
& +\int_{t_{0}}^{t} \psi^{\prime}(s)(\psi(t)-\psi(s))^{\alpha-1} \mathbb{E}_{\alpha, \alpha}\left(\mathbf{A}(\psi(t)-\psi(s))^{\alpha}\right) \odot f(s) \odot d s .
\end{aligned}
$$

3. Linear Systems. Consider the linear fractional dynamical system governed by $\psi$-Hilfer fractional differential equation of the form

$$
\begin{aligned}
\mathbb{H}_{\oplus, \odot, t_{0}+}^{\alpha, \beta ; \psi} x(t) & =A x(t) \oplus B u(t), \quad t \in\left[t_{0}, t_{1}\right] \\
\mathbb{I}_{\oplus, \odot, t_{0}+}^{1-\gamma ; \psi} x\left(t_{0}\right) & =0
\end{aligned}
$$

with $0<\alpha<1,0 \leq \beta \leq 1, \gamma=\alpha+\beta(1-\alpha)$, the state vector $x \in \mathbb{R}^{n}$, the control vector $u \in \mathbb{R}^{m}$ and $A$ and $B$ are the constant matrices of dimension $n \times n$ and $n \times m$ respectively. The solution representation of (27) is

$x(t)=\int_{\left[t_{0}, t\right]}^{\oplus} g^{-1}\left(\psi^{\prime}(s)(\psi(t)-\psi(s))^{\alpha-1}\right) \odot \mathbb{E}_{\alpha, \alpha}\left(g(A)(\psi(t)-\psi(s))^{\alpha}\right) \odot B \odot u(s) \odot \mathrm{ds}$.

Clearly, $x\left(t_{0}\right)=0$.

Definition 3.1 (Reachable). The linear system (27) is called reachable in time $t_{1}$ if for each vector $x_{1} \in \mathbb{R}^{n}$ there exists an input function $u(t) \in L^{2}\left(\left[t_{0}, t_{1}\right] ; \mathbb{R}^{n}\right)$, which steers the state of the system (27) from the zero initial state $x\left(t_{0}\right)=0$ to the final state $x\left(t_{1}\right)=x_{1}$.

Theorem 3.2 (Reachability Grammian). The system (27) is reachable in time $t \in\left[t_{0}, t_{1}\right]$ if and only if the "Reachability Grammain"

$R\left(t_{0}, t_{1}\right)=\int_{\left[t_{0}, t_{1}\right]}^{\oplus} \mathbb{E}_{\alpha, \alpha}\left(g(A)(\psi(t)-\psi(s))^{\alpha}\right) \odot \mathbf{B} \odot \mathbf{B}^{*} \odot \mathbb{E}_{\alpha, \alpha}\left(g\left(A^{*}\right)(\psi(t)-\psi(s))^{\alpha}\right) \odot \mathrm{ds}$

is positive definite.

Proof. Suppose the reachability Grammian $R\left(t_{0}, t_{1}\right)$ is positive definite, then define the input function

$u(t)=\left[g^{-1}\left(\psi^{\prime}(t)\left(\psi\left(t_{1}\right)-\psi(t)\right)^{\alpha-1}\right)\right]^{-1} \odot \mathbf{B}^{*} \odot \mathbb{E}_{\alpha, \alpha}\left(g\left(\mathbf{A}^{*}\right)\left(\psi\left(t_{1}\right)-\psi(t)\right)^{\alpha}\right) \odot R^{-1}\left(t_{0}, t_{1}\right) \odot x_{1}$. 
Using (28), (29) and (30), we obtain

$$
\begin{aligned}
x\left(t_{1}\right)= & \int_{\left[t_{0}, t_{1}\right]}^{\oplus} g^{-1}\left(\psi^{\prime}(s)\left(\psi\left(t_{1}\right)-\psi(s)\right)^{\alpha-1}\right) \odot \mathbb{E}_{\alpha, \alpha}\left(g(\mathbf{A})\left(\psi\left(t_{1}\right)-\psi(s)\right)^{\alpha}\right) \odot \mathbf{B} \\
& \odot\left[g^{-1}\left(\psi^{\prime}(s)\left(\psi\left(t_{1}\right)-\psi(s)\right)^{\alpha-1}\right)\right]^{-1} \odot \mathbf{B}^{*} \odot \mathbb{E}_{\alpha, \alpha}\left(g\left(A^{*}\right)\left(\psi\left(t_{1}\right)-\psi(s)\right)^{\alpha}\right) \odot R^{-1}\left(t_{0}, t_{1}\right) \odot x_{1} \odot \mathrm{ds} \\
= & \int_{\left[t_{0}, t_{1}\right]}^{\oplus} g^{-1}\left(\psi^{\prime}(s)\left(\psi\left(t_{1}\right)-\psi(s)\right)^{\alpha-1}\right) \odot\left[g^{-1}\left(\psi^{\prime}(s)\left(\psi\left(t_{1}\right)-\psi(s)\right)^{\alpha-1}\right)\right]^{-1} \\
& \odot \mathbb{E}_{\alpha, \alpha}\left(g(\mathbf{A})\left(\psi\left(t_{1}\right)-\psi(s)\right)^{\alpha}\right) \odot \mathbf{B} \odot \mathbf{B}^{*} \odot \mathbb{E}_{\alpha, \alpha}\left(g\left(\mathbf{A}^{*}\right)\left(\psi\left(t_{1}\right)-\psi(s)\right)^{\alpha}\right) \odot \mathrm{ds} \odot \mathrm{R}^{-1}\left(\mathrm{t}_{0}, \mathrm{t}_{1}\right) \odot \mathrm{x}_{1} \\
= & \int_{\left[t_{0}, t_{1}\right]}^{\oplus} \mathbb{E}_{\alpha, \alpha}\left(g(\mathbf{A})\left(\psi\left(t_{1}\right)-\psi(s)\right)^{\alpha}\right) \odot \mathbf{B} \odot \mathbf{B}^{*} \odot \mathbb{E}_{\alpha, \alpha}\left(g\left(\mathbf{A}^{*}\right)\left(\psi\left(t_{1}\right)-\psi(s)\right)^{\alpha}\right) \odot \mathrm{ds} \odot \mathrm{R}^{-1}\left(\mathrm{t}_{0}, \mathrm{t}_{1}\right) \odot \mathrm{x}_{1} \\
= & R\left(t_{0}, t_{1}\right) \odot R^{-1}\left(t_{0}, t_{1}\right) \odot x_{1} \\
= & x_{1} .
\end{aligned}
$$

Therefore, the input function (30) steers the state of the system (27) from 0 to $x_{1}$.

Suppose the reachability Grammian $R\left(t_{0}, t_{1}\right)$ is not positive definite, then there exists a non-zero $z$ such that $z^{*} \odot R\left(t_{0}, t_{1}\right) \odot z=0$. It means that

$z^{*} \odot \int_{\left[t_{0}, t_{1}\right]}^{\oplus} \mathbb{E}_{\alpha, \alpha}\left(g(\mathbf{A})(\psi(t)-\psi(s))^{\alpha}\right) \odot \mathbf{B} \odot \mathbf{B}^{*} \odot \mathbb{E}_{\alpha, \alpha}\left(g\left(\mathbf{A}^{*}\right)(\psi(t)-\psi(s))^{\alpha}\right) \odot \mathrm{ds} \odot \mathrm{z}=0$.

This implies

$$
z^{*} \odot \mathbb{E}_{\alpha, \alpha}\left(g(\mathbf{A})(\psi(t)-\psi(s))^{\alpha}\right) \odot \mathbf{B}=0 \text { on }\left[t_{0}, t_{1}\right] .
$$

From the assumption, there exist an input function $u(t)$ such that, the state of the system steers from origin to $z$ in $\left[t_{0}, t_{1}\right]$. It follows that

$x\left(t_{1}\right)=z=\int_{\left[t_{0}, t_{1}\right]}^{\oplus} g^{-1}\left(\psi^{\prime}(s)\left(\psi\left(t_{1}\right)-\psi(s)\right)^{\alpha-1}\right) \odot \mathbb{E}_{\alpha, \alpha}\left(g(\mathbf{A})\left(\psi\left(t_{1}\right)-\psi(s)\right)^{\alpha}\right) \odot \mathbf{B} \odot u(s) \odot \mathrm{d} \mathbf{s}$.

Then

$z^{*} \odot z=z^{*} \odot \int_{\left[t_{0}, t_{1}\right]}^{\oplus} g^{-1}\left(\psi^{\prime}(s)\left(\psi\left(t_{1}\right)-\psi(s)\right)^{\alpha-1}\right) \odot \mathbb{E}_{\alpha, \alpha}\left(g(\mathbf{A})\left(\psi\left(t_{1}\right)-\psi(s)\right)^{\alpha}\right) \odot \mathbf{B} \odot u(s) \odot \mathrm{ds}$.

Since the right hand side term is zero and conclude that $z^{*} \odot z=0$. This leads to a contradiction that $z \neq 0$. Thus $R\left(t_{0}, t_{1}\right)$ is positive definite.

4. Non-linear Systems. Consider the nonlinear fractional dynamical system governed by $\psi$-Hilfer fractional differential equation of the form

$$
\begin{aligned}
\mathbb{H}_{\oplus, \odot, t_{0}+}^{\alpha, \beta ; \psi} x(t) & =\mathbf{A} x(t) \oplus \mathbf{B} u(t) \oplus f(t, x(t), u(t)), \quad t \in\left[t_{0}, t_{1}\right] \\
\mathbb{I}_{\oplus, \odot, t_{0}+}^{1-\gamma} x\left(t_{0}\right) & =0
\end{aligned}
$$

with $0<\alpha<1,0 \leq \beta \leq 1, \gamma=\alpha+\beta(1-\alpha)$, the state vector $x \in \mathbb{R}^{n}$, the control vector $u \in \mathbb{R}^{m}$ and $\mathbf{A}$ and $\mathbf{B}$ are the constant matrices of dimension $n \times n$ and $n \times m$ respectively and the nonlinear function $f:\left[t_{0}, t_{1}\right] \times \mathbb{R}^{n} \times \mathbb{R}^{m} \rightarrow \mathbb{R}^{n}$ is continuous.

Let us introduce the following notations: Denote as $X$ the Banach space of continuous $\mathbb{R}^{n} \times \mathbb{R}^{m}$ value functions defined on the interval $\left[t_{0}, t_{1}\right]$ with the norm $\|(x, u)\|=\|x\|+\|u\|$, where $\|x\|=\sup \left\{|x(t)|: t \in\left[t_{0}, t_{1}\right]\right\}$ and $\|u\|=\sup \{|u(t)|:$ $\left.t \in\left[t_{0}, t_{1}\right]\right\}$. 
I2 VANTERLER DA C. SOUSA, M. VELlAPPANDI, V. GOVINDARAJ AND GASTÃO S. F. FREDERICO.

For each $(y, w) \in X$, consider the linear system

$$
\begin{aligned}
& \mathbb{H}_{\oplus, \odot, t_{0}+}^{\alpha, \beta ; \psi} x(t)=\mathbf{A} x(t) \oplus \mathbf{B} u(t) \oplus f(t, y(t), w(t)), \quad t \in\left[t_{0}, t_{1}\right] \\
& \mathbb{I}_{\oplus, \odot, t_{0}+}^{1-\gamma ; \psi} x\left(t_{0}\right)=0 .
\end{aligned}
$$

Then the solution is given by

$$
\begin{aligned}
x(t)= & \int_{\left[t_{0}, t\right]}^{\oplus} g^{-1}\left(\psi^{\prime}(s)(\psi(t)-\psi(s))^{\alpha-1}\right) \odot \mathbb{E}_{\alpha, \alpha}\left(g(\mathbf{A})(\psi(t)-\psi(s))^{\alpha}\right) \odot \mathbf{B} \odot u(s) \odot \mathrm{ds} \\
& \oplus \int_{\left[t_{0}, t\right]}^{\oplus} g^{-1}\left(\psi^{\prime}(s)(\psi(t)-\psi(s))^{\alpha-1}\right) \odot \mathbb{E}_{\alpha, \alpha}\left(g(\mathbf{A})(\psi(t)-\psi(s))^{\alpha}\right) \odot f(s, y(s), w(s)) \odot \mathrm{ds} .
\end{aligned}
$$

Let assume the following:

$[\mathbf{H}]$ : The continuous function $f$ satisfies the condition

$$
\lim _{|(x, u)| \rightarrow \infty} \frac{|f(t, x, u)|}{|(x, u)|}=0
$$

uniformly in $t \in\left[t_{0}, t_{1}\right]$.

Theorem 4.1. Suppose the linear system (27) is reachable and the assumption [H] holds, then the nonlinear system (31) is reachable on $\left[t_{0}, t_{1}\right]$.

Proof. Define the operator $T: X \rightarrow X$ by

$$
T(y, w)=(x, u)
$$

where

$$
\begin{aligned}
u(t)= & {\left[g^{-1}\left(\psi^{\prime}(t)\left(\psi\left(t_{1}\right)-\psi(t)\right)^{\alpha-1}\right)\right]^{-1} \odot \mathbf{B}^{*} \odot \mathbb{E}_{\alpha, \alpha}\left(g\left(\mathbf{A}^{*}\right)\left(\psi\left(t_{1}\right)-\psi(t)\right)^{\alpha}\right) \odot R^{-1}\left(t_{0}, t_{1}\right) } \\
& \odot\left[x_{1} \ominus \int_{\left[t_{0}, t_{1}\right]}^{\oplus} g^{-1}\left(\psi^{\prime}(s)\left(\psi\left(t_{1}\right)-\psi(s)\right)^{\alpha-1}\right) \odot \mathbb{E}_{\alpha, \alpha}\left(g(\mathbf{A})\left(\psi\left(t_{1}\right)-\psi(s)\right)^{\alpha}\right) \odot f(s, y(s), w(s)) \odot \mathrm{d} s\right]
\end{aligned}
$$

and

$$
\begin{aligned}
x(t)= & \int_{\left[t_{0}, t\right]}^{\oplus} g^{-1}\left(\psi^{\prime}(s)(\psi(t)-\psi(s))^{\alpha-1}\right) \odot \mathbb{E}_{\alpha, \alpha}\left(g(\mathbf{A})(\psi(t)-\psi(s))^{\alpha}\right) \odot \mathbf{B} \odot u(s) \odot \mathrm{ds} \\
& \oplus \int_{\left[t_{0}, t\right]}^{\oplus} g^{-1}\left(\psi^{\prime}(s)(\psi(t)-\psi(s))^{\alpha-1}\right) \odot \mathbb{E}_{\alpha, \alpha}\left(g(\mathbf{A})(\psi(t)-\psi(s))^{\alpha}\right) \odot f(s, y(s), w(s)) \odot \mathrm{ds} .
\end{aligned}
$$

Let

$$
\begin{aligned}
\tilde{a}_{1} & =\left\|g^{-1}\left(\psi^{\prime}(t)\left(\psi\left(t_{1}\right)-\psi(t)\right)^{\alpha-1}\right)\right\| \\
\tilde{a}_{2} & =\left\|\mathbb{E}_{\alpha, \alpha}\left(g(\mathbf{A})\left(\psi\left(t_{1}\right)-\psi(t)\right)^{\alpha}\right)\right\| \\
\sup |f| & =\sup \left\{|f(t, y(t), w(t))|: t \in\left[t_{0}, t_{1}\right]\right\} \\
\tilde{d} & =4\left[\frac{1}{\tilde{a}_{1}} \odot\left\|\mathbf{B}^{*}\right\| \odot \tilde{a}_{2} \odot\left\|R^{-1}\left(t_{0}, t_{1}\right)\right\| \odot\left|x_{1}\right|\right] \\
\tilde{a} & =\sup \left\{\tilde{a}_{1} \odot \tilde{a}_{2} \odot\|\mathbf{B}\|\left(t_{1}-t_{0}\right), 1\right\} \\
\tilde{c}_{1} & =4\left[\left\|\mathbf{B}^{*}\right\| \odot \tilde{a}_{2}^{2} \odot\left\|R^{-1}\left(t_{0}, t_{1}\right)\right\|\left(t_{1}-t_{0}\right)\right] \\
\tilde{c}_{2} & =4\left[\tilde{a}_{1} \odot \tilde{a}_{2}\left(t_{1}-t_{0}\right)\right] \\
\tilde{c} & =\max \left\{\tilde{c}_{1}, \tilde{c}_{2}\right\} .
\end{aligned}
$$


Then

$$
\begin{aligned}
|u(t)| & \leq \frac{1}{\tilde{a}_{1}} \odot\left\|\mathbf{B}^{*}\right\| \odot \tilde{a}_{2} \odot\left\|R^{-1}\left(t_{0}, t_{1}\right) \odot\right\|\left[\left|x_{1}\right| \odot \int_{\left[t_{0}, t\right]}^{\oplus} \tilde{a}_{1} \odot \tilde{a}_{2} \odot \sup |f| \odot \mathrm{ds}\right] \\
& \leq \frac{\tilde{d}}{4 \tilde{a}} \oplus \frac{\tilde{c}}{4 \tilde{a}} \odot \sup |f| \\
& \leq \frac{\tilde{d}}{4 \tilde{a}} \oplus \frac{\tilde{c}}{4 \tilde{a}} \odot \sup |f| \leq \frac{\tilde{d}}{4 \tilde{a}}+\frac{\tilde{c}}{4 \tilde{a}} \sup |f|, \text { for all g } \cdot \\
|x(t)| & \leq \int_{\left[t_{0}, t\right]}^{\oplus} \tilde{a}_{1} \odot \tilde{a}_{2} \odot\|B\| \odot\|u\| \odot \operatorname{ds} \oplus \int_{\left[\mathrm{t}_{0}, \mathrm{t}\right]}^{\oplus} \tilde{\mathrm{a}}_{1} \odot \tilde{a}_{2} \odot \sup |\mathrm{f}| \odot \mathrm{ds} \\
& \leq \tilde{a}_{1} \odot \tilde{a} \tilde{a}_{2} \odot\|\mathbf{B}\| \odot\|u\| \odot\left(t_{1}-t_{0}\right) \oplus \tilde{a}_{1} \odot \tilde{a}_{2} \odot \sup |f| \odot\left(t_{1}-t_{0}\right) \\
& \leq \tilde{a} \odot\|u\| \oplus \frac{\tilde{c}_{2}}{4} \odot \sup |f| \\
& \leq \frac{\tilde{d}}{4} \oplus \frac{\tilde{c}}{4} \odot \sup |f| \oplus \frac{\tilde{c}}{4} \odot \sup |f| \\
& \leq \frac{\tilde{d}}{2} \oplus \frac{\tilde{c}}{2} \odot \sup |f| \leq \frac{\tilde{d}}{2}+\frac{\tilde{c}}{2} \sup |f|, \text { for all g } \cdot
\end{aligned}
$$

By the Theorem [12], for each pair of positive constants $\tilde{c}$ and $\tilde{d}$, there exists a positive constant $\tilde{r}$ such that, if $\|q\| \leq r$ then

$$
\tilde{c}|f(t, q)|+\tilde{d} \leq \tilde{r} \text { for all } t \in\left[t_{0}, t_{1}\right]
$$

If $\|y\| \leq \frac{\tilde{r}}{2}$ and $\|w\| \leq \frac{\tilde{r}}{2}$, then $|y(t)|+|w(t)| \leq \tilde{r}$, for all $t \in\left[t_{0}, t_{1}\right]$. It follows that $\tilde{d}+\tilde{c} \sup |f| \leq \tilde{r}$. Therefore, $|u(t)| \leq \frac{\tilde{r}}{4 \tilde{a}}$ for all $t \in\left[t_{0}, t_{1}\right]$ and hence $\|u\| \leq \frac{\tilde{r}}{4 \tilde{a}}$ which gives $\|x\| \leq \frac{\tilde{r}}{2}$. Thus we have proved that if $X(\tilde{r})=\left\{(y, w):\|y\| \leq \frac{\tilde{r}}{2}\right.$ and $\|w\| \leq$ $\left.\frac{\tilde{r}}{2}\right\}$ then $T$ maps $X(\tilde{r})$ into itself. Since $f$ is continuous, this implies that the operator is continuous and hence is completely continuous by the application of Arzela-Ascoli theorem. Since $X(\tilde{r})$ is closed, bounded and convex, the Schauder fixed point theorem guarantees that $T$ has a fixed point $(y, w) \in X(r)$ such that $T(y, w)=(y, w)=(x, u)$. Hence, we have

$$
\begin{aligned}
x(t)= & \int_{\left[t_{0}, t\right]}^{\oplus} g^{-1}\left(\psi^{\prime}(s)(\psi(t)-\psi(s))^{\alpha-1}\right) \odot \mathbb{E}_{\alpha, \alpha}\left(g(\mathbf{A})(\psi(t)-\psi(s))^{\alpha}\right) \odot \mathbf{B} \odot u(s) \odot \mathrm{ds} \\
& \oplus \int_{\left[t_{0}, t\right]}^{\oplus} g^{-1}\left(\psi^{\prime}(s)(\psi(t)-\psi(s))^{\alpha-1}\right) \odot \mathbb{E}_{\alpha, \alpha}\left(g(\mathbf{A})(\psi(t)-\psi(s))^{\alpha}\right) \odot f(s, y(s), w(s)) \odot \mathrm{ds} .
\end{aligned}
$$

Then, $x(t)$ is the solution of the system (31) and $x\left(t_{1}\right)=x_{1}$. Hence the system (31) is reachable on $\left[t_{0}, t_{1}\right]$.

\section{Numerical Results.}

Example 5.1. Consider the linear fractional dynamical system described by $\psi$ Hilfer fractional differential equation as

$$
\begin{aligned}
\underset{\oplus, \odot, 0+}{\mathbb{H}_{\stackrel{3}{4}, \frac{1}{2} ; t^{2}+t}} & =\left[\begin{array}{ll}
1 & 2 \\
0 & 2
\end{array}\right] x(t) \oplus\left[\begin{array}{l}
0 \\
1
\end{array}\right] u(t), \quad t \in[0,2] \\
\mathbb{I}_{\oplus, \odot, 0+}^{\frac{1}{8} ; t^{2}+t} x(0) & =0 .
\end{aligned}
$$


I4 VANTERLER DA C. SOUSA, M. VELLAPPANDI, V. GOVINDARAJ AND GASTÃO S. F. FREDERICO.

Comparing with (27), we have $\mathbf{A}=\left[\begin{array}{ll}1 & 2 \\ 0 & 2\end{array}\right], \mathbf{B}=\left[\begin{array}{l}0 \\ 1\end{array}\right], x(0)=\left[\begin{array}{l}0 \\ 0\end{array}\right]$ and $x(t)=\left[\begin{array}{l}x_{1}(t) \\ x_{2}(t)\end{array}\right]$. Let $g(t)=t^{2}$ and let us consider the final state vector $x(1)=$ $\left[\begin{array}{l}2 \\ 1\end{array}\right]$. The Mittag-Leffler matrix function for the matrix $g(\mathbf{A})=\mathbf{A}^{2}$ is

$$
\mathbb{E}_{3 / 4,3 / 4}\left(\mathbf{A}^{2}\left(\left(t^{2}+t\right)-\left(s^{2}+s\right)\right)^{3 / 4}\right)=\left[\begin{array}{cc}
K_{1} & -2 K_{1}+2 K_{2} \\
0 & K_{2}
\end{array}\right],
$$

where $K_{1}=\mathbb{E}_{3 / 4,3 / 4}\left(\left(\left(t^{2}+t\right)-\left(s^{2}+s\right)\right)^{3 / 4}\right)$ and $K_{2}=\mathbb{E}_{3 / 4,3 / 4}\left(4\left(\left(t^{2}+t\right)-\left(s^{2}+s\right)\right)^{3 / 4}\right)$.

The reachability Grammian matrix for this system is

$$
\begin{aligned}
R(0,2) & =\int_{0}^{2} \mathbb{E}_{3 / 4,3 / 4}\left(\mathbf{A}^{2}\left(6-\left(s^{2}+s\right)\right)^{3 / 4}\right) \mathbf{B B}^{*} \mathbb{E}_{3 / 4,3 / 4}\left(\mathbf{A}^{* 2}\left(6-\left(s^{2}+s\right)\right)^{3 / 4}\right) \mathrm{ds} \\
& =\left[\begin{array}{cc}
13509583798.5227 & 6768523781.6375 \\
6768523781.6375 & 3391141901.4305
\end{array}\right]>0 .
\end{aligned}
$$

Hence, the system (34) is reachable on $[0,2]$. It means that, the input function

$$
u(t)=\frac{\left(6-\left(t^{2}+t\right)\right)^{1 / 4}}{(2 t+1)^{1 / 2}} \mathbf{B}^{*} \mathbb{E}_{3 / 4,3 / 4}\left(\mathbf{A}^{* 2}\left(6-\left(t^{2}+t\right)\right)^{3 / 4}\right) R^{-1}(0,2) x_{1}
$$

transfer the states of the system (34) from the initial point $x(0)=\left[\begin{array}{l}0 \\ 0\end{array}\right]$ to the final point $x(2)=\left[\begin{array}{l}2 \\ 1\end{array}\right]$ during the time interval $[0,2]$.

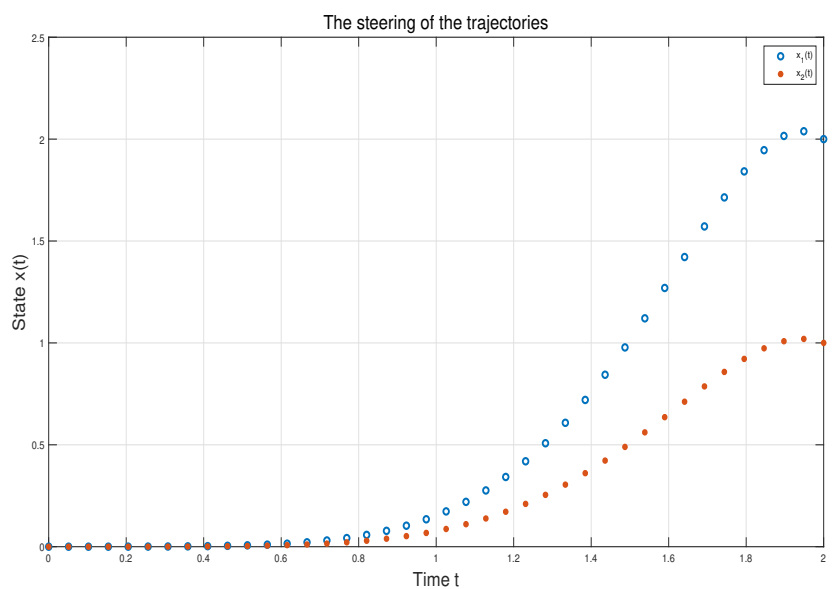

Fig. 1. The trajectory of the system (34) steers from initial point $\left[\begin{array}{l}0 \\ 0\end{array}\right]$ to the final point $\left[\begin{array}{l}2 \\ 1\end{array}\right]$ during the interval $[0,2]$. 




Fig. 2. The steering input function $u(t)$ of the system (34) during the interval $[0,2]$.

Example 5.2. Consider the nonlinear fractional dynamical system described by $\psi$-Hilfer fractional differential equation as

$$
\begin{aligned}
\mathbb{H}_{\oplus, \odot, 0+}^{\frac{1}{2}, \frac{3}{4} ; t^{3}+2 t} & =\left[\begin{array}{ll}
0 & 1 \\
1 & 0
\end{array}\right] x(t) \oplus\left[\begin{array}{l}
0 \\
1
\end{array}\right] u(t) \oplus f(t, x(t), u(t)), \quad t \in[0,1] \\
\mathbb{I}_{\oplus, \odot, 0+}^{\frac{1}{8} ; t^{3}+2 t} x(0) & =0 .
\end{aligned}
$$

Comparing with (32), we have $\mathbf{A}=\left[\begin{array}{ll}0 & 1 \\ 1 & 0\end{array}\right], \mathbf{B}=\left[\begin{array}{l}0 \\ 1\end{array}\right], x(0)=\left[\begin{array}{l}0 \\ 0\end{array}\right], x(t)=$ $\left[\begin{array}{l}x_{1}(t) \\ x_{2}(t)\end{array}\right]$ and $f(t, x(t), u(t))=\left[\begin{array}{c}\sin x_{1}(t) \\ \cos u(t)\end{array}\right]$. Let $g(t)=t^{3}$ and let us consider the final state vector $x(1)=\left[\begin{array}{l}4 \\ 6\end{array}\right]$. The Mittag-Leffler matrix function for the matrix $g(\mathbf{A})=\mathbf{A}^{3}$ is

$$
\mathbb{E}_{1 / 2,1 / 2}\left(\mathbf{A}^{3}\left(\left(t^{3}+2 t\right)-\left(s^{3}+2 s\right)\right)^{1 / 2}\right)=\left[\begin{array}{cc}
N_{1} & N_{2} \\
N_{2} & N_{1}
\end{array}\right]
$$

where

$N_{1}=\frac{1}{2}\left[\mathbb{E}_{1 / 2,1 / 2}\left(-\left(\left(t^{3}+2 t\right)-\left(s^{3}+2 s\right)\right)^{1 / 2}\right)+\mathbb{E}_{1 / 2,1 / 2}\left(\left(\left(t^{3}+2 t\right)-\left(s^{3}+2 s\right)\right)^{1 / 2}\right)\right]$

and

$N_{2}=\frac{1}{2}\left[\mathbb{E}_{1 / 2,1 / 2}\left(\left(\left(t^{3}+2 t\right)-\left(s^{3}+2 s\right)\right)^{1 / 2}\right)-\mathbb{E}_{1 / 2,1 / 2}\left(-\left(\left(t^{3}+2 t\right)-\left(s^{3}+2 s\right)\right)^{1 / 2}\right)\right]$.

The corresponding reachability Grammian matrix for this system is

$$
\begin{aligned}
R(0,1) & =\int_{0}^{1} \mathbb{E}_{1 / 2,1 / 2}\left(\mathbf{A}^{3}\left(\left(t^{3}+2 t\right)-\left(s^{3}+2 s\right)\right)^{1 / 2}\right) \mathbf{B B}^{*} \mathbb{E}_{1 / 2,1 / 2}\left(\mathbf{A}^{* 3}\left(\left(t^{3}+2 t\right)-\left(s^{3}+2 s\right)\right)^{1 / 2}\right) \mathrm{ds} \\
& =\left[\begin{array}{ll}
86.1384 & 88.5424 \\
88.5424 & 91.1055
\end{array}\right]>0
\end{aligned}
$$

and the given nonlinear function satisfies the Assumption $[H]$. Hence, by the Theorem (4.1) the nonlinear system (35) is reachable on $[0,1]$. The input vector and 
I6 VANTERLER DA C. SOUSA, M. VELlAPPANDI, V. GOVINDARAJ AND GASTÃO S. F. FREDERICO.

state trajectory pair $(x(t), u(t))$ can be approximated by iterate $u^{(n)}(t)$ defined by

$$
\begin{aligned}
u^{(n+1)}(t)= & \frac{\left(3-\left(t^{3}+2 t\right)\right)^{1 / 6}}{\left(3 t^{2}+2\right)^{1 / 3}} \mathbf{B}^{*} \mathbb{E}_{1 / 2,1 / 2}\left(\mathbf{A}^{* 3}\left(3-\left(t^{3}+2 t\right)\right)^{1 / 2}\right) R^{-1}(0,1) \\
& \times\left[x_{1}-\int_{0}^{1}\left(\left(3 s^{2}+2\right)\left(3-\left(s^{3}+2 s\right)\right)^{1 / 2}\right)^{1 / 3} \mathbb{E}_{1 / 2,1 / 2}\left(\mathbf{A}^{3}\left(3-\left(s^{3}+2 s\right)\right)^{1 / 2}\right)\right. \\
& \left.\times f\left(s, x^{(n)}(s), u^{(n)}(s)\right) \mathrm{d} s\right]
\end{aligned}
$$

and the state vector approximated $x^{(n)}(t)$ at $n^{t h}$ stage is in turn given by the approximated scheme $\left\{x_{j}^{(n)}\right\}$ defined as

$$
\begin{aligned}
x_{j+1}^{(n)}(t)= & \int_{0}^{t}\left(\left(3 s^{2}+2\right)\left(\left(t^{3}+2 t\right)-\left(s^{3}+2 s\right)\right)^{1 / 2}\right)^{1 / 3} \mathbb{E}_{1 / 2,1 / 2}\left(\mathbf{A}^{3}\left(\left(t^{3}+2 t\right)-\left(s^{3}+2 s\right)\right)^{1 / 2}\right) \\
& \times\left(\mathbf{B} u^{(n)}(s)+f\left(s, x^{(n)}(s), u^{(n)}(s)\right)\right) \mathrm{d} s .
\end{aligned}
$$

The state trajectories $x(t)$ and steering input function $u(t)$ are computed and are depicted in Figure $\mathbf{3}$ and Figure $\mathbf{4}$ respectively.



Fig. 3. The trajectory of the system (35) steers from initial point $\left[\begin{array}{l}0 \\ 0\end{array}\right]$ to the final point $\left[\begin{array}{l}4 \\ 6\end{array}\right]$ during the interval $[0,1]$. 


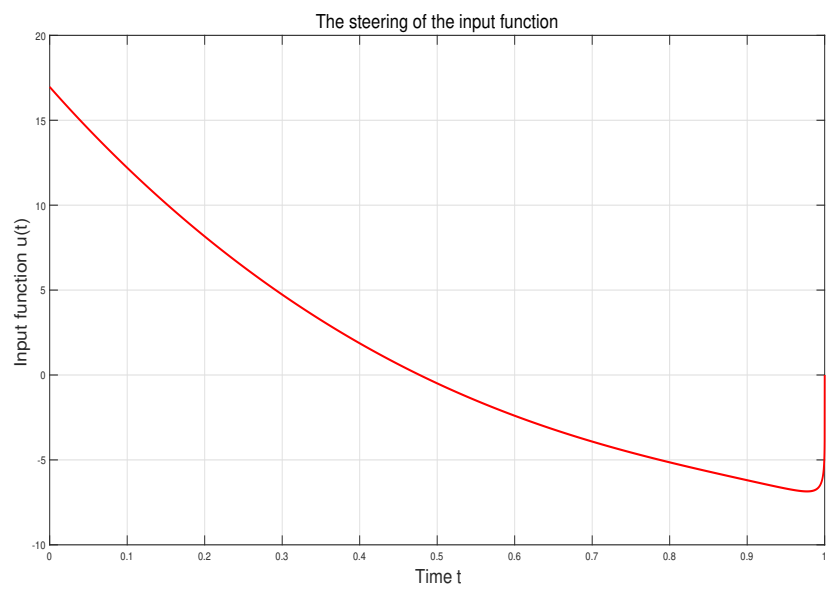

Fig. 4. The steering input function $u(t)$ of the system (35) during the interval $[0,1]$.

\section{REFERENCES}

[1] A. Babakhani, M. Yadollahzadeh, A. Neamaty, Some properties of pseudo-fractional operators, J. Pseudo-Diff. Oper. Appl., 9 (3) (2018), 677-700.

[2] K. Balachandran, J.Y. Park, J.J. Trujillo, Controllability of nonlinear fractional dynamical systems, Nonlinear Analysis: Theory, Methods $\&$ Applications 75 (2012), 1919-1926.

[3] K. Balachandran, V. Govindaraj, L. Rodríguez-Germá, Juan J. Trujillo, Controllability of nonlinear higher order fractional dynamical systems, Nonlinear Dynamics 71 (2013), 605612.

[4] K. Balachandran, V. Govindaraj, L. Rodríguez-Germá, Juan J. Trujillo, Controllability results for nonlinear fractional-order dynamical systems, J. Optimization Theory Appl. 156 (2013), 33-44.

[5] K. Balachandran, V. Govindaraj, L. Rodríguez-Germá, Juan J. Trujillo, Stabilizability of fractional dynamical systems, Fractional Calculus \& Applied Analysis, 17 (2014), 511-531.

[6] K. Balachandran, V. Govindaraj, M. Rivero, Juan J. Trujillo, Controllability of fractional damped dynamical systems, Applied Mathematics and Computation, 257 (2015), 66-73.

[7] P. Borisut, P. Kumam, I. Ahmed, W. Jirakitpuwapat, Existence and uniqueness for $\psi$-Hilfer fractional differential equation with nonlocal multi-point condition, Math. Meth. Appl. Sci., (2020), https://doi.org/10.1002/mma.6092.

[8] A. Boudjerida, D. Seba, and G. M. N'Guérékata, Controllability of coupled systems for impulsive $\phi$-Hilfer fractional integro-differential inclusions, Applicable Anal., (2020), 1-18.

[9] M. Buslowicz, Controllability, reachability and minimum energy control of fractional discretetime linear systems with multiple delays in state, Bull. Polish Academy Sci. Tech. Sci., 62(2) (2014), 233-239.

[10] E. Capelas de Oliveira, J. Vanterler da C. Sousa, Ulam-Hyers-Rassias stability for a class of fractional integro-differential equations, Results Math., 73 (3) (2018), 111.

[11] Q. Dai, R. Gao, Z. Li, C. Wang, Stability of Ulam-Hyers and Ulam-Hyers-Rassias for a class of fractional differential equations, Adv. Diff. Equ., 2020(1) (2020), 1-15.

[12] J.P. Dauer, Nonlinear perturbations of quasi-linear control problems, J. Math. Anal. Appl., 54 (1976), 717-725.

[13] Y. Do Ri, H.C. Choi, K. J. Chang, Constructive Existence of Solutions of Multi-point Boundary Value Problem for Hilfer Fractional Differential Equation at Resonance, Mediterr. J. Math., 17 (95) (2020).

[14] V. Govindaraj, K. Balachandran, Stability of Basset equation, Journal of Fractional Calculus and Applications, 5 (2014), 1-15.

[15] V. Govindaraj, Raju K. George, Controllability of fractional dynamical systems: A functional analytic approach, Math. Control \& Related Fields, 7 (4) (2017), 537-562.

[16] T. L. Guo, Controllability and observability of impulsive fractional linear time-invariant system, Comput. Math. Appl., 64 (10) (2012), 3171-3182. 
I8 VANTERLER DA C. SOUSA, M. VELLAPPANDI, V. GOVINDARAJ AND GASTÃO S. F. FREDERICO.

[17] M. Hosseini, A. Babakhani, H. Agahi, S.H. Rasouli,. On pseudo-fractional integral inequalities related to Hermite-Hadamard type, Soft Computing, 20 (7) (2016), 2521-2529.

[18] T. Kaczorek, Fractional positive continuous-time linear systems and their reachability, Int. J. Appl. Math. Comput. Sci, 18(2) (2008), 223-228.

[19] T. Kaczorek, Reachability of positive 2D fractional linear systems, Physica Scripta, (2009), 1621-1631.

[20] T. Kaczorek, Reachability of cone fractional continuous-time linear systems, Int. J. Appl. Math. Comput. Sci., 19(1) (2009), 89-93.

[21] T. Kaczorek, Reachability of fractional positive continuous-time linear systems, J. Automation, Mobile Robotics 83 Intelligent Systems, 3 (2009), 1-7.

[22] T. Kaczorek, Positivity and reachability of fractional electrical circuits, Acta mechanica et automatica, 5 (2011), 42-51.

[23] T. Kaczorek, Minimum energy control of fractional descriptor positive discrete-time linear systems with bounded inputs, The International Federation of Automatic Control, 47(3) (2014), 2909-2914.

[24] T. Kaczorek, Reachability Of fractional continuous-time linear systems using the CaputoFabrizio derivative $30^{\text {th }}$ European Conference on Modelling and Simulation, (2016), 53-58.

[25] T. Kaczorek, Relationships between the reachability of positive standard and fractional discrete-time and continuous-time linear systems, Adv. Intelligent Sys. Comput., 577 (2017), 401-414.

[26] T. Kaczorek, Reachability and observability of the fractional linear systems with state and output feedbacks, Automation, 743 (2018), 116-131.

[27] J. P. Kharade, Kishor D. Kucche, On the impulsive implicit $\psi$-Hilfer fractional differential equations with delay, Math. Meth. Appl. Sci., 43(4) (2020), 1938-1952.

[28] R. Kociszewski, Minimum energy control of fractional discrete-time linear systems with delays in state and control, Recent Advances in Automation, Robotics and Measuring Techniques, 267 (2014), 127-136.

[29] Z. Liu, X. Li, J. Sun, Controllability of nonlinear fractional impulsive evolution systems, J. Integral Equ. Appl., 25 (2013), 395-406.

[30] D. Mozyrska, E. Pawłuszewicz, M. Wyrwas, Local observability and controllability of nonlinear discrete-time fractional order systems based on their linearisation, Inter. J. Sys. Sci., 48 (2017), 788-794.

[31] E. Pap, Pseudo-additive measures and their applications, Handbook of measure theory, NorthHolland, 2002, 1403-1468.

[32] E. Pap, Applications of the generated pseudo-analysis to nonlinear partial differential equations, Contemporary Math., 377 (2005), 239-260.

[33] L. Sajewski, Reachability, observability and minimum energy control of fractional positive continuous-time linear systems with two different fractional orders, Multidim Syst Sign Process, 27(1) (2016), 27-41.

[34] K. Rogowski, Reachability of standard and fractional continuous-time systems with constant inputs, Archives of Control Sci., 26(2) (2016),147-159.

[35] W. Trzasko, Reachability and controllability of positive fractional discrete-time systems with delay, Journal of Automation, Mobile Robotics and Intelligent Systems, 2(3) (2008).

[36] J. Vanterler da C. Sousa, E. Capelas de Oliveira, On the $\psi$-Hilfer fractional derivative, Commun. Nonlinear Sci. Numer. Simul., 60 (2018), 72-91.

[37] J. Vanterler da C. Sousa, E. Capelas de Oliveira, Leibniz type rule: $\psi$-Hilfer fractional operator, Commun. Nonlinear Sci. Numer. Simul. 77 (2019), 305-311.

[38] J. Vanterler da C. Sousa, E. Capelas de Oliveira, Kishor D. Kucche, On the fractional functional differential equation with abstract Volterra operator, Bull. Braz. Math. Soc. New Series, 50 (4) (2019), 803-822.

[39] J. Vanterler da C. Sousa, Kishor D. Kucche, E. Capelas De Oliveira, Stability of $\psi$-Hilfer impulsive fractional differential equations, Appl. Math. Lett., 88 (2019), 73-80.

[40] J. Vanterler da C. Sousa, Mouffak Benchohra, Gaston M. N'Guérékata, Attractivity for differential equations of fractional order and $\psi$-Hilfer type, Fract. Calc. Appl. Anal., 23 (4) (2020), $1188-1207$.

[41] J. Vanterler da C. Sousa, G.S.F. Frederico, E. Capelas de Oliveira, $\psi$-Hilfer pseudofractional operator: new results about fractional calculus, Comput. Appl. Math., https://doi.org/10.1007/s40314-020-01304-6 (2020). 
[42] J. Wang, Y. Zhou, Complete controllability of fractional evolution systems, Commun. Nonlinear Sci. Numer. Simul., 17(11) (2012), 4346-4355.

[43] D. Xu, Y. Li, W. Zhou, Controllability and Observability of Fractional Linear Systems with Two Different Orders, The Scientific World Journal, Article ID 618162, (2014), 1-8.

[44] H. Zhang, J. Cao, and W. Jiang, Reachability and controllability of fractional singular dynamical systems with control delay, J. Appl. Math., 2013(1) (2013), 1-10.

Received xxxx 20xx; revised xxxx 20xx.

E-mail address: vanterlermatematico@hotmail.com

E-mail address: vellappandim@gmail.com

E-mail address: govindaraj .maths@gmail.com

E-mail address: gastao.frederico@ua.pt 